Revue internationale de l'économie sociale

Recma

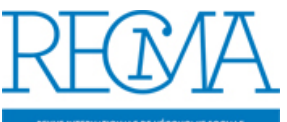

\title{
La coopération agricole en Grèce : changements structurels et adaptations au contexte de crise
}

\section{Simeon Karafolas et Androniki Katarachia}

Numéro 329, juillet 2013

URI : https://id.erudit.org/iderudit/1017937ar

DOI : https://doi.org/10.7202/1017937ar

Aller au sommaire du numéro

Éditeur(s)

Association Recma

ISSN

1626-1682 (imprimé)

2261-2599 (numérique)

Découvrir la revue

Citer cet article

Karafolas, S. \& Katarachia, A. (2013). La coopération agricole en Grèce :

changements structurels et adaptations au contexte de crise. Revue

internationale de l'économie sociale, (329), 108-115.

https://doi.org/10.7202/1017937ar 


\section{LA COOPÉRATION AGRICOLE EN GRÈCE : CHANGEMENTS STRUCTURELS ET ADAPTATIONS AU CONTEXTE DE CRISE}

par Simeon Karafolas et Androniki Katarachia*

\author{
*Institut d'éducation techno- \\ logique de Macédoine de \\ l'Ouest, Kozani, Grèce. \\ Mél. : skarafolas@yahoo.gr. \\ Texte traduit de l'anglais \\ par Patricia Toucas-Truyen.
}

La coopération agricole constitue la forme coopérative la plus répandue en Grèce et l'un des principaux mouvements coopératifs agricoles en Europe, avec 6545 entités enregistrées à la fin de l'année 2012. Structurée de façon pyramidale, la coopération agricole grecque repose au premier degré sur les structures communales, dont les membres sont des personnes physiques. Au second degré, les cent douze unions de coopératives agricoles fonctionnent à l'échelon préfectoral. Enfin, des organisations coopératives de troisième degré couvrent certains produits ou groupes de produits au niveau national. Presque toutes les coopératives sont membres de la Confédération panhellénique des unions de coopératives agricoles (Paseges).

$\mathrm{Au}$ cours des dernières années, les coopératives agricoles grecques ont dû s'adapter à des changements structurels importants liés aux conséquences de la crise économique. Ces modifications découlent essentiellement du nouveau cadre législatif voté en 2011, mais aussi de l'acquisition de la Banque agricole de Grèce par une banque commerciale, la Banque du Pirée. La restructuration vise à réorganiser la coopération agricole sur la base de la responsabilité entrepreneuriale. La crise économique a impacté les ressources financières de ces entreprises: elles ont tenté d'y répondre par des innovations dans la production, par la promotion de leurs produits et en créant de nouvelles activités.

\section{L'ÉVOLUTION HISTORIQUE DE LA LÉGISLATION}

La réglementation sur les coopératives agricoles grecques a évolué au gré des orientations que les gouvernements successifs souhaitaient donner à celles-ci. Jusqu'à la fin des années 80 , les coopératives étaient sous l'influence des interventions de l'Etat et des partis politiques. Durant la décennie 90, il est devenu évident qu'elles devaient fonctionner avec des critères d'intérêt privé. La législation, dont le point d'orgue est la loi de 2011 sur les coopératives agricoles, a alors essayé de les doter de plus d'autonomie et d'un caractère entrepreneurial. Plusieurs périodes peuvent être distinguées jusqu'au vote de cette loi. 


\section{Le rôle de 1'Etat}

En 1914, a été promulguée la première loi sur les organisations coopératives (loi 602-1914). L'Etat a exercé sa supervision par le biais du ministère des Finances nationales, jusqu'à la création en 1917 du ministère de l'Agriculture, qui a ensuite assumé cette responsabilité. Cette loi est restée en vigueur jusqu'à son remplacement par la loi 921-79, en 1979.

Entre les deux guerres, l'Etat a commencé à entraver le fonctionnement des coopératives en tentant de les instrumentaliser. La réforme agraire qui s'est déroulée entre 1923 et 1932 a en effet été confrontée à l'établissement des réfugiés en provenance des territoires occupés par l'armée turque en Asie Mineure. Elle a modifié le fonctionnement économique des petites exploitations familiales dans le pays et a accompagné l'essor des coopératives agricoles, qui ont joué un rôle primordial dans le développement économique de la population. Au tournant des années 30, les gouvernements ont tenté de mettre en œuvre un plan ambitieux, en établissant des coopératives obligatoires exclusivement dévolues à la réalisation de projets productifs dans des zones rurales. Fin 1932, quinze coopératives de ce type avaient été établies et cinquante et une autres étaient en voie de constitution. Le projet gigantesque pour le développement des terres agricoles en Grèce du Nord s'appuyait largement sur ces coopératives, qui relevaient de la loi 4639-1930. A la même période, en 1929, la Banque agricole de Grèce (ATE) a été fondée afin d'aider le secteur. Elle est devenue la principale source de financements et d'informations pour les agriculteurs et la plupart des coopératives agricoles. Celles-ci se sont regroupées en 1936 au sein de la Confédération panhellénique des unions de coopératives agricoles (Paseges), qui, suspendue pendant la guerre, a été rétablie en 1943 sous la loi 479-1943.

De 1959 à 1974, la fonction des coopératives a été affectée par la dictature des colonels. Les assemblées générales se sont vu retirer leurs pouvoirs, qui ont été transférés à des conseils d'administration désignés par le gouvernement dictatorial. Les coopératives rurales ont, de fait, été dissoutes.

\section{Marché commun et banalisation}

Entre 1974 et 1980, il a fallu redéfinir la fonction organisationnelle des coopératives. L'accession de la Grèce au marché commun européen a accéléré le besoin d'un nouveau cadre juridique. La loi 921-1979 visait à moderniser les coopératives et à les intégrer en tant qu'entreprises dans l'économie nationale.

Entre 1981 et 1989, elles se sont politisées et, dans une large mesure, identifiées au parti politique au pouvoir, le parti socialiste (Pasok). Cette loi a rénové l'organisation structurelle du mouvement et imposé de nouvelles règles de fonctionnement, comme la fusion obligatoire des coopératives afin qu'il n'en subsiste qu'une dans chaque municipalité ou communauté et qu'il n'y ait qu'une seule union de coopératives par préfecture. De plus, la loi a établi pour la première fois la collaboration entre coopératives et entreprises privées, à condition que la participation de la coopérative au capital excède $65 \%$.

De 1990 à la nouvelle législation de 2011, il est devenu évident que les coopératives devaient fonctionner sur des critères d'entreprise privée. Durant cette période, plusieurs lois ont été votées, pour certaines avec une cible 
spécifique, comme la loi sur les dettes des coopératives envers la Banque agricole de Grèce (ATE). Progressivement, les membres et les employés des coopératives ont obtenu le droit d'acquérir des parts coopératives optionnelles sans droit de vote. La direction pouvait être confiée par le conseil d'administration à un dirigeant non membre.

Les coopératives sont restées sous la tutelle du ministre de l'Agriculture, qui pouvait déléguer leur supervision à une autre personne ou autorité. Les organisations coopératives agricoles de n'importe quel niveau ou les entreprises coopératives pouvaient établir des partenariats avec des entreprises privées. Des incitations à la fusion étaient octroyées sous forme de soutien financier et d'exemption fiscale.

\section{LA NOUVELLE LOI DE RÉORGANISATION DE L'AGRICULTURE}

En septembre 2011, a été votée la loi 4015-2011 «sur la structure des coopératives agricoles, des organisations collectives et de l'entrepreneuriat dans le secteur agricole ». Le secteur (essentiellement coopératif) a dû affronter les changements les plus importants de son histoire, au moins sur le plan législatif.

Ces modifications ont eu des répercussions sur l'organisation de la coopération et le nombre de coopératives. La nouvelle réglementation résulte du constat que l'intervention politique en faveur des coopératives agricoles, en agissant sur leur fonctionnement, a provoqué de sérieux problèmes dans l'administration et le financement, notamment les subventions publiques provenant de l'Union européenne et de l'Etat grec. Fondée sur la responsabilité entrepreneuriale des coopératives, elle réclame en outre, l'exercice d'une surveillance plus étroite par une autorité de tutelle, la création d'un registre national pour les organisations agricoles et une évaluation périodique.

La nouvelle loi prévoit une rénovation structurelle reposant sur les coopératives agricoles primaires. Celles de second degré sont supprimées et le rôle de Paseges est drastiquement limité. Cette structure a été renforcée par l'introduction de deux formes d'organisation qui vont dans le sens de la responsabilité entrepreneuriale des coopératives. L'une, les partenariats ruraux, met en relation des coopératives agricoles et des groupes de producteurs. L'autre, les organisations collectives rurales, rassemble des coopératives agricoles de premier degré, des groupes de producteurs et des partenariats ruraux.

Les modifications réglementaires concernaient le nombre des coopératives, mais également les formes coopératives du secteur agricole. On estime globalement qu'il y a 6500 coopératives agricoles, parmi lesquelles, selon le ministre de l'Agriculture, un millier seulement présentent un chiffre d'affaires positif. En outre, 5200 coopératives seraient des «unions fantômes". Avec les nouveaux critères très stricts qui leur seront appliqués, nombre de coopératives agricoles et de groupes de producteurs n'existeront plus dans un futur proche. Les plus touchées seront les coopératives de second degré, qui n'ont pas directement d'activité de production. Les coopératives ont jusqu'à mi-2013 (après prolongations) pour fusionner et montrer qu'elles sont actives, soit dans la production agricole, soit dans l'emballage et l'exportation. Le registre national des organisations agricoles collectives 
constitue l'outil central d'évaluation, en les classant en deux catégories: les actives et les inactives. Elles seront inspectées une fois par an et elles seront supprimées si elles ne présentent aucun signe d'activité pendant trois années consécutives. Les coopératives ont plus que jamais l'obligation de travailler comme des entreprises dans une économie de marché, une condition préalable pour participer aux nouvelles organisations interprofessionnelles.

\section{Trois types d'organisation agricole collective}

La nouvelle loi permet l'établissement de trois formes d'organisation agricole collective, pour lesquelles elle offre des incitations fiscales au développement: coopératives agricoles, groupes de producteurs et partenariats d'entreprises agricoles.

\section{Les coopératives agricoles}

Il peut s'agir de coopératives de pêche (maritimes ou piscicoles), d'élevage de bétail ou de volaille, d'apiculture, de sériculture, agro-tourisme, etc. Pour s'établir, elles doivent réunir au minimum vingt membres et présenter un capital social d'au moins 60000 euros. Quatre-vingts pour cent de la production des membres doivent être détenus par la coopérative. Cette obligation, très stricte, est tout à fait inédite.

\section{Les groupes de production}

Il s'agit d'associations de producteurs agricoles, qui peuvent être établies par des coopératives agricoles ou des producteurs individuels. Ces groupes organisent la production de certains produits, notamment dans le secteur agroalimentaire. Ils fournissent intrants et matériel et mettent en place des services de normalisation, le stockage, la vente et la commercialisation des produits agricoles.

\section{Les partenariats d'entreprises agricoles}

D'une part, il existe des partenariats agriculture-entreprise, qui sont des sociétés anonymes (SA) et des sociétés à responsabilité limitée (SARL) actives à l'échelle interdisciplinaire et intersectorielle. Elles engagent des activités commerciales au niveau national et régional sur la production,

(1) Les entreprises civiles sont des sociétés créées par deux ou plusieurs personnes, physiques ou morales, afin de menerdes activités communes à caractère économique ou social. Cette forme est utilisée pourdes activitésscientifiques, charitables, économiques ou commerciales. Par exemple, une société civile peut être créée pour la mise en œuvre des programmes communautaires liés à l'intégration économique et sociale des groupes sociaux défavorisés. l'emballage, le stockage, la transformation industrielle et la commercialisation des produits agricoles.

D'autre part, les partenariats agroalimentaires sont des entreprises civiles ${ }^{(1)}$ à but non lucratif, qui peuvent être fondées à l'initiative des autorités régionales. L'objectif est de valoriser les produits agricoles régionaux. Participent à l'établissement de ces entreprises les municipalités de la région, les organisations collectives agricoles, les établissements industriels ou commerciaux, touristiques, de restauration ou de consommation (personnes physiques et morales) qui sont en activité dans la région et dans le secteur des produits agricoles alimentaires ou encore les universités et les centres de recherche régionaux qui travaillent dans des disciplines et des domaines de recherche en rapport avec le projet initial. 


\section{DES INCITATIONS FISCALES À LA CROISSANCE}

La loi favorise le développement des organisations collectives agricoles (CAO) par des allègements fiscaux. Elles peuvent bénéficier d'une aide financière par le biais du fonds pour l'agriculture et l'élevage afin de mettre en œuvre leurs programmes et de promouvoir leurs produits. Elles peuvent être financées durant la première année de l'opération jusqu'à l'approbation par les services compétents de l'Union européenne pour couvrir la totalité ou une partie des coûts d'installation et d'opération. La loi envisage également la possibilité d'incitation à la fusion, au développement et à la conversion des coopératives agricoles figurant au registre national. Elle prévoit que les statuts des coopératives agricoles et les amendements soient exemptés de droit de timbre ou de toute autre taxation en faveur de l'Etat ou d'un tiers. Les avantages fiscaux liés aux fusions sont ainsi appliqués aux organisations coopératives agricoles. De plus, l'acquisition d'un bien auprès de l'une d'elles en vue de développer une activité économique est soumise au même régime fiscal favorable que si l'Etat en était l'acquéreur.

\section{Les enchères de produits agricoles}

La loi 4015-11 introduit pour la première fois les enchères de produits agricoles afin de réduire l'écart entre les prix des producteurs et les prix à la consommation. Celles-ci sont organisées et gérées comme des sociétés anonymes (SA), sous la surveillance du ministère du Développement rural et de l'Alimentation. Il ne peut y en avoir plus de deux par région (la Grèce est divisée en treize régions). Leur capital social est géré par des organisations agricoles collectives, les autorités régionales et locales compétentes, des personnes individuelles et le ministre du Développement rural et de l'Alimentation, qui représente l'Etat. Les organisations collectives agricoles avec les autorités régionales et locales doivent détenir au moins $51 \%$ du capital social des ventes aux enchères. Cela leur permet d'avoir le contrôle de chaque opération. Les producteurs privés et les coopératives peuvent écouler leurs produits par le biais de la salle de vente et signer des contrats de longue durée pour la vente de leur production aux enchères avec des conditions et des garanties convenues ou qui ont été déterminées préalablement par la maison des enchères. Les coopératives agricoles participant à une salle de vente sont tenues d'écouler leur production par son intermédiaire.

\section{L'acquisition de la Banque agricole de Grèce (ATE)}

La Banque agricole de Grèce (ATE), fondée en 1929, a étéla principale institution pour l'application de la politique agricole du pays en finançant, en organisant et en contrôlant les coopératives. Son rôle a été celui d'une banque coopérative Raiffeisen dans une zone rurale. L'ATE a financé des coopératives agricoles avec des taux d'intérêt privilégiés, et elle a parfois effacé des dettes, qui dans certains cas ont été reprises par l'Etat (par les lois 2198-1994 et 2538-1997, par exemple). En tant que principale source de financement, elle a accordé aux agriculteurs et aux coopératives des prêts hypothécaires dont la valeur excédait les pratiques habituelles des banques, allant jusqu’à $120 \%$ de la valeur nominale 
des emprunts. On estime qu'environ $70 \%$ de la zone agricole est hypothéquée, principalement auprès de l'ATE.

En 2012, la Banque de Grèce a décidé le rappel de la licence d'exploitation de l'ATE et a nommé un mandataire $a d$ hoc. Il s'en est suivi la division de l'ATE en deux structures, le " mauvais ATE » ayant des actifs douteux et le «bon ATE » ayant des actifs sains. En juillet 2012, le comité des mesures de réorganisation à la Banque de Grèce (la banque centrale grecque) a décidé de transférer les bons actifs et les passifs à la Banque du Pirée. Ce transfert signe l'arrêt de mort de l'ATE et met fin en même temps aux liens privilégiés de celle-ci avec le monde agricole, et notamment les coopératives agricoles. Ces dernières se retrouvent dans un nouvel environnement, où elles sont considérées comme n'importe quelle entreprise pour l'accès au système financier. Le problème le plus urgent à résoudre est celui des dettes que les coopératives ont, soit auprès du «mauvais ATE » (quand ces dettes sont liées aux emprunts non remboursés), soit auprès de la Banque du Pirée, pour les emprunts effectués normalement. L'un des aspects de ce problème est la fin du crédit-bail comme outil financier pour la création de stations photovoltaïques, que les coopératives agricoles avaient souscrit ces dernières années.

\section{LES COOPÉRATIVES AGRICOLES FACE À LA CRISE}

Bien que l'agriculture n'ait pas subi la même récession que d'autres secteurs comme le bâtiment, les coopératives ont dû faire face à une conjoncture économique difficile. Une partie de la production agricole est exportée et une autre est consommée en Grèce. L'impact de la baisse de la consommation sur le marché national a été plutôt limité, en comparaison avec celui des produits de luxe, pour la plupart importés, comme les véhicules. L'impact a été assez indirect, provenant de clients en prise avec de sérieuses difficultés pour rembourser leurs dettes à leurs créanciers, y compris les coopératives agricoles. L'effet domino qui est apparu dans l'économie grecque a eu des répercussions sur nombre de secteurs, comprenant l'agriculture et les coopératives agricoles.

\section{Liquidités et dévaluation immobilière}

Les coopératives agricoles grecques rencontrent des problèmes de liquidités, comme la plupart des acteurs économiques, qu'il s'agisse des banques, des

(2) Les chèques différés, de quelques jours à quelques mois, sont une pratique commune en Grèce dans le commerce solidaire. En fait, ils sont plus souvent utilisés comme prêt du fournisseur à son client. Ils sont habituellement acceptés par les banques comme une enchère pour offrir un prêt de fonds de roulement aux entreprises. entreprises ou des simples consommateurs. Du fait du manque de liquidité général, elles ne parviennent pas, elles qui sont généralement payées par des chèques différés, à se faire rembourser ${ }^{(2)}$. Une autre conséquence importante de la dévaluation du prix de l'immobilier pour les sociétés agricoles est la perte de valeur de leur propriété hypothéquée, notamment auprès de l'ATE (acquise auprès de la Banque du Pirée). Au cours de la période 2010-2012, la baisse cumulée des prix des appartements en Grèce, excepté à Athènes et à Thessalonique, est estimée à $20 \%$. Pour de vieilles usines et des entrepôts, la chute est encore plus importante, dans la mesure où ces propriétés sont désaffectées. Par conséquent, les coopératives agricoles grecques se retrouvent face à des problèmes financiers 
considérables. Si nous examinons un échantillon de cinquante unions de coopératives agricoles, nous observons une croissance du nombre des chèques impayés et des lettres de créance par ces coopératives allant de 216 en 2010 à 356 en 2011 et 809 en 2012 (cette année-là, la valeur des obligations non payées a été de 46,5 millions d'euros) ${ }^{(3)}$.

(3) Y compris les propriétés immobilières confisquées aux coopératives. Source : Icap Business Service Group. Lettreàl'auteur,19février2013.

\section{Investissements productifs}

Malgré les difficultés financières et économiques, les coopératives agricoles grecques se sont lancées dans des investissements productifs, pour différencier leurs produits et être plus compétitives dans ce contexte de crise. Elles ont également investi dans leur réseau de vente, en créant de nouvelles formes de vente, surtout par le commerce sur Internet. Un grand nombre d'entre elles se sont intéressées à la production d'énergie durable de type photovoltaïque ou éolienne.

Les investissements ont été engagés par des coopératives sur plusieurs produits agricoles, notamment dans l'agroalimentaire, au sein de toutes les régions grecques, pour améliorer la production et la qualité du produit. Des investissements importants ont été réalisés sur la production d'huile d'olive dans le processus de transformation. A titre d'exemple, des investissements de plus de 2,5 millions d'euros ont été faits au cours de cette période par trois coopératives agricoles dans le Péloponnèse, les îles Ioniennes, les îles de la mer Egée, pour la production d'huile d'olive. D'autres de plus de 5 millions d'euros ont été faits par dix coopératives agricoles en Macédoine centrale, concernant les fruits (surtout les kiwis) et les pommes de terre. En Thessalie, une coopérative vinicole a investi dans l'unité de production d'un spécial « tsipouro ». Deux coopératives des régions de Thessalie et de l'Epire ont programmé des investissements très importants - jusqu'à 46 millions d'euros - dans la production de volaille et de bétail.

Les coopératives ont également investi dans le commerce électronique. Ainsi, certaines ont créé un réseau Internet pour stimuler les ventes de fruits en Macédoine occidentale et de vin et d'huile dans le Péloponnèse.

Durant la crise, le développement de sources d'énergie alternatives est devenu une activité intéressante, en partie à cause des prix subventionnés pour les producteurs d'énergie, comme le photovoltaïque. Ces mesures ont intéressé de nombreux agriculteurs et des coopératives qui étaient en passe d'acquérir des terrains disponibles. Paseges a informé les coopératives locales sur la possibilité de créer des stations photovoltaïques et éoliennes en coopération avec le ministère de l'Environnement. Ces initiatives ont incité une coopérative crétoise à investir dans l'énergie éolienne pour plus de 3 millions d'euros et une coopérative du Péloponnèse à engager plus de 1 million sur le photovoltaïque.

\section{CONCLUSION}

Pendant la crise, les coopératives agricoles grecques ont dû affronter, comme n'importe quelle entreprise, les conséquences économiques et financières de la récession, du manque de liquidités du système bancaire et des difficultés 
rencontrées par les acteurs économiques, qu'ils soient des individus ou des sociétés. Elles ont été affectées par ces problèmes économiques et financiers, comme en témoignent le non-paiement des obligations financières, les chèques non payés et les lettres de créance.

Mais en même temps, les nombreuses coopératives agricoles ont investi et pris des initiatives qui témoignent d'un dynamisme entrepreneurial. La nouvelle loi sur l'organisation de l'agriculture et l'acquisition de la Banque agricole de Grèce a induit des modifications structurelles importantes, qui impactent comme jamais auparavant le caractère des coopératives agricoles, désormais fondé sur la philosophie de l'entreprise. Elles doivent fonctionner avec des critères d'intérêt privé, sans le filet de sécurité de la Banque agricole de Grèce et sans l'appui de l'Etat. 